\title{
Using the full turbulence spectrum for describing droplet coalescence and breakage in industrial liquid-liquid systems: experiments and modeling
}

\author{
Simone Castellano ${ }^{1,2}$, Lorenzo Carrillo ${ }^{1}$, Nida Sheibat-Othman ${ }^{2}$, Daniele Marchisio ${ }^{3}$, Antonio \\ Buffo $^{3}$, Sophie Charton ${ }^{1} *$ \\ ${ }^{1}$ CEA, DEN, Research Department on Mining and Fuel Recycling Processes, SA2I, FR-30207, \\ Bagnols-sur-Cèze, France \\ ${ }^{2}$ Université Claude Bernard Lyon 1,CNRS, UMR 5007, LAGEP, 43 bd 11 Novembre 1918, FR- \\ 69622 Villeurbanne, France \\ ${ }^{3}$ Politecnico di Torino, Dipartimento di Scienza Applicate e Tecnologie, Corso Duca degli Abruzzi \\ 24, IT-10129, Torino, Italy \\ * Corresponding author: sophie.charton@cea.fr
}

\begin{abstract}
A generalized model for breakage and coalescence kernels valid for the entire spectrum of turbulence is proposed and validated. Most of the available kernels in the literature, indeed, assume that in a turbulent liquid-liquid dispersion, the dispersed droplets have dimension in the inertial subrange and are affected by eddies with size in the same subrange. These kernels are based on the Kolmogorov second-order structure function, which is valid only in the inertial subrange. However, in most industrially encountered situations, many droplets may have a size in the dissipation range, where the Kolmogorov second-order structure function does not apply. Therefore, a more general description of the energy transferred between these droplets and the turbulent eddies is needed to properly model breakage and coalescence events.

In this work, the Coulaloglou and Tavlarides breakage and coalescence kernels [1] will be modified through the implementation of the second-order structure function proposed by Davidson [2], along with the Pope energy spectrum [3]. Turbulent liquid-liquid
\end{abstract}


dispersion experiments at high continuous phase viscosity are performed to test and validate the model. The generalized model is able to predict the experimental Sauter mean diameters at different viscosities, turbulent conditions and dispersed-phase volume fraction without any adjustment of the kernel parameters.

\section{Keywords:}

Turbulent liquid-liquid dispersion, breakage and coalescence kernel, second-order structure function, energy spectrum, Kolmogorov microscale, viscous continuous phase.

\section{Introduction}

Many processes in the chemical, pharmaceutical, oil and food industries are based on turbulent liquid-liquid dispersions. These flows are characterized by the presence of a continuous phase in which droplets of a second immiscible phase are dispersed. It is the case of solvent extraction processes, where high mass-transfer efficient separation requires a high interfacial surface.

In order to describe the change in time of the interfacial area, a Population Balance Equation (PBE) $[4,5]$ could be employed. The PBE is an integral-differential equation that computes the time-evolution of the number density function (NDF), which represents the distribution of the droplet population with regards to some properties, called internal variables (size, solute concentration, temperature, etc.). Considering the droplet size as internal coordinate, the interfacial area is directly tracked.

The PBE model includes sub-models in the source terms, called kernels, whose formulation allows to physically describe the evolution of the NDF. The number of droplets breaking or coalescing per unit time is computed through these kernels. Both the breakage and coalescence kernels strongly depend on the physical-chemical properties of the liquids and on the flow characteristics. Indeed, on one hand, in the turbulent flow, the 
breakage and coalescence mechanisms are intrinsically related to the drop-eddy interactions [6-9]. Smaller eddies transmit their turbulent kinetic energy to droplets, that are hence likely to break [10], whereas bigger ones transport the droplets in the flow, increasing their probability to collide and coalesce [11]. The kernels will be therefore strongly dependent on the turbulent kinetic energy dissipation rate $\varepsilon$, which represents the energy dissipated by the eddies. On the other hand, the droplet breakage and coalescence are influenced by the dispersed and continuous phase viscosities and densities, and the surface tension.

For turbulent liquid-liquid dispersions, many studies have dealt with the effect of the dispersed phase viscosity on droplet breakage (see e.g.[12-14]). The authors stated that in order to achieve breakage, the turbulent dissipation rate must not only be higher than the droplet surface energy, but it should also overcome the sum of the latter and the droplet viscous energy, which is proportional to the internal viscous stresses that oppose to droplet deformation. On the contrary, few studies have considered the effect of the continuous phase viscosity on the statistical properties of the turbulent flow that influence droplet breakage and coalescence [15-17]. Indeed, most of the kernels presented in the literature assume that the droplets have dimension in the inertial subrange of turbulence. According to the Kolmogorov second similarity hypothesis [3], in this subrange, the statistical properties of the turbulent flow depend on the turbulent dissipation rate $\varepsilon$ and are independent of the continuous phase viscosity; therefore, this property was not considered in the kernel formulation.

However, in a liquid-liquid dispersion, it is frequent that droplets exhibit dimensions of different orders of magnitude (from microns to millimeters). For this reason, a nonnegligible number of droplets is likely to fall outside the inertial subrange, i.e. in the 
dissipation subrange (Fig. 1). In this case, the statistical properties of the turbulent flow depend both on the turbulent dissipation rate and on the continuous phase viscosity.

Therefore, employing a kernel which assumes the droplets to be in the inertial subrange is not valid, since the properties of the turbulent flow, and especially the turbulence kinetic energy, can be also affected by the viscosity of the continuous phase. In addition, evaluating the turbulent kinetic energy of an eddy in the dissipation subrange using the Kolmogorov energy spectrum, valid for the inertial subrange only, leads to an overestimation of the eddy energy, since the dampening effect of viscosity is not considered.

Following the recent exploratory studies of Karimi and Andersson [15], based on the review of turbulence theory development of Solsvik and Jakobsen [16], the objective of this work is to formulate a set of coalescence and breakage kernel functions in which the effect of the continuous phase viscosity on the turbulence is accounted for. In this aim, a general second-order structure function is computed [2], derived from a full eddy energy spectrum [3]. The latter energy spectrum accounts not only for the eddy energy distribution in both the inertial and the dissipative ranges, but also in the energy containing range, where the turbulence is anisotropic. Moreover, experimental measurements are carried out in order to test and validate the proposed modifications.

Regarding breakage and coalescence, the Coulaloglou and Tavlarides models [1], which were found appropriate for continuous phases with low viscosity [18], have been kept as the basis and modified to account a wider energy spectrum. A OD PBE model was employed in order to reduce the computation time, where the turbulent inhomogeneity in the liquid-liquid contactor is considered using the method proposed by Buffo et al. [19] and recently adopted with success by Castellano et al. [20] for the prediction of droplet size distributions in a mechanically agitated liquid-liquid contactor. This method is based 
on the computation of volume-average kernels through the volumetric probability density function of the turbulent dissipation rate estimated by CFD simulations.

To test the modified Coulaloglou and Tavlarides kernels, liquid-liquid dispersion experiments were performed using different continuous phases with increasing viscosities, to mimic the possible variations encountered in extracting systems. Higher continuous phase viscosity leads to a wider dissipation subrange, and therefore to a higher probability of finding droplets belonging to this viscosity-dependent energy range.

The experimental values of the Sauter mean diameter were compared with the predictions of the Coulaloglou and Tavlarides kernels using i) the "generalized" formulation proposed here and $\mathrm{ii}$ ) assuming that the droplets size belongs to the inertial subrange only using the original model, indicated in the text as "initial formulation" (model used in [20]).

\section{Model development}

\subsection{Improved second-order structure function}

Most of the kernels proposed in literature rely on the Kolmogorov theory [3]. This theory assumes local isotropy of turbulence and is based on two similarity hypotheses. The local isotropy assumption states that for a very high Reynolds number, the geometrical and directional information of the biggest eddies is lost in the cascade process. The first similarity hypothesis assumes the statistical properties of the turbulent flow to be dependent only on the turbulent dissipation rate, $\varepsilon$, and on the continuous phase kinematic viscosity, $v$, at scales smaller than the characteristic length of the flow $(r \ll L)$. Additionally, according to the second similarity hypothesis, for scales much bigger than the Kolmogorov microscale $(r \gg \eta)$, the statistical properties of the turbulent flow depend only on $\varepsilon$. The Kolmogorov scale represents the smallest size an eddy can have in a turbulent flow before being dissipated: 


$$
\eta=\left(\frac{v^{3}}{\varepsilon}\right)^{\frac{1}{4}}
$$

The interval in which the local isotropy hypothesis applies (isotropic turbulence) is called the universal range. It can be subdivided into two subranges: the inertial and the dissipation subranges [2,3]. Both similarity hypotheses apply in the inertial subrange, while only the first one is valid in the dissipation subrange. The boundary $\left(l_{D I}\right)$ between the two subranges has been established by Pope [3]:

$$
l_{D I} \approx 60 \eta \text {. }
$$

Using dimensional analysis, Kolmogorov expressed the second-order structure function valid for the inertial subrange, as:

$$
\left\langle[\Delta u(r)]^{2}\right\rangle=C_{S} \varepsilon^{\frac{2}{3}} r^{\frac{2}{3}}
$$

$\left\langle[\Delta u(r)]^{2}\right\rangle$ represents the square of the average relative velocity between two points at distance $r$ in the turbulent flow. This function is of fundamental importance in the definition of breakage and coalescence kernels. Indeed, as will be highlighted in Section 2.2, both the eddy turbulent kinetic energy and the droplets collision frequency will be computed considering $r=d$. It is important to remark that Eq. (3), established by Kolmogorov by applying the two similarity hypotheses, does not involve the continuous phase viscosity.

Most of the kernels proposed in the literature address the coalescence and breakage of droplets having dimensions in the same range as the characteristic turbulent eddy size in the inertial subrange. This means that the size of the droplets involved in the turbulent liquid-liquid dispersion must obey: $d>l_{D I}$ (the lower limit of the inertial subrange). However, it is possible that a significant number of the dispersed droplets do not have size in this subrange, and that the turbulent stresses to which they are subjected depend both on $\varepsilon$ and $v$. If so, the assumption of a second-order structure function according to Eq. (3) in 
the breakage definition could lead to erroneous breakage and coalescence rates. Indeed, when this function is applied to the dissipation range, it overestimates the eddy energy, since this latter tends to zero due to viscosity dissipation effects (Fig. 1).

As a result, a refined formulation for the second-order structure function is needed to predict correctly the droplet breakage and coalescence. Davidson [2] proposed a secondorder structure function $\left\langle[\Delta u(r)]^{2}\right\rangle$ which is based on the cumulative contribution of the turbulent kinetic energy of the eddies of size smaller than $r$ and of the enstrophy of eddies of size larger than $r$ :

$$
\begin{aligned}
\left\langle[\Delta u(r)]^{2}\right\rangle= & (\text { turbulent kinetic energy of eddies with size }<r) \\
& +(\text { enstrophy of eddies with size }>r)
\end{aligned}
$$

The author expressed this function as $[2,15,16]$ :

$$
\left\langle[\Delta u(r)]^{2}\right\rangle=\frac{4}{3} \int_{0}^{\infty} E(\kappa)+3 E(\kappa)\left[\frac{\cos (\kappa r)}{(\kappa r)^{2}}-\frac{\sin (\kappa r)}{(\kappa r)^{3}}\right] d \kappa
$$

Eq. (4) requires the knowledge of the energy spectrum $E(\kappa)$, which gives the distribution of the turbulent kinetic energy across eddies of different sizes. In this work, the model proposed by Pope [3], for the whole energy spectrum, was employed:

$$
E(\kappa)=C \varepsilon^{\frac{2}{3}} \kappa^{-\frac{5}{3}}\left(\frac{\kappa L}{\left[(\kappa L)^{2}+c_{L}\right]^{\frac{1}{2}}}\right)^{\frac{11}{3}} \exp \left(-\beta\left\{\left[(\kappa \eta)^{4}+c_{\eta}^{4}\right]^{\frac{1}{4}}-c_{\eta}\right\}\right)
$$

where the parameters $C=1.5, \beta=5.2$ have been experimentally determined [2,21] and $L=\frac{k^{\frac{3}{2}}}{\varepsilon}[3] . c_{\eta}, c_{L}$ are retrieved by enforcing the following definitions:

$$
\left\{\begin{array}{c}
\varepsilon-\int_{0}^{\infty} 2 v \kappa^{2} E\left(\kappa, c_{L}, c_{\eta}\right) d \kappa=0 \\
k-\int_{0}^{\infty} E\left(\kappa, c_{L}, c_{\eta}\right) d \kappa=0
\end{array}\right.
$$


The Pope energy spectrum function accounts for both the energy-containing range, where the turbulence is anisotropic, and the universal range (that combines the inertial and dissipation subranges).

\subsection{Breakage and Coalescence kernels}

Following the definition of a more general expression for the second-order structure function in Sec. 2.1, the Coulaloglou and Tavlarides kernels can be modified computing $\left\langle[\Delta u(d)]^{2}\right\rangle$ according to Eq. (4). This means that the mechanism responsible for droplet breakage and coalescence is still due to turbulence fluctuations, but the range of the interacting eddies now includes eddies of all characteristic sizes.

The breakage kernel expresses the fraction of droplets with diameter $d$ breaking in a unit time:

$$
\Gamma(d)=\frac{1}{t_{b}} \frac{N_{\text {break }}(d)}{N_{\text {tot }}(d)}
$$

The breakage time $t_{b}$ is proportional to the separation velocity of two lumps of fluid in a turbulent flow [22]:

$$
t_{b}=\frac{d}{\sqrt{\left\langle[\Delta u(d)]^{2}\right\rangle}}
$$

Coulaloglou and Tavlarides assumed that breakage occurs when a droplet interacts with an eddy of smaller or equal size, which has a turbulent kinetic energy higher than the droplet surface energy:

$$
\frac{N_{\text {break }}(d)}{N_{\text {tot }}(d)}=e^{-\frac{E_{\text {surface,droplet }}(d)}{E_{\text {kinetic }, e d d y}(d)}}
$$

where:

$$
\begin{gathered}
E_{\text {surface,droplet }}(d)=k_{1} \sigma d^{2} \\
E_{\text {kinetic,eddy }}(d)=k_{2} \rho_{d} d^{3}\left\langle[\Delta u(d)]^{2}\right\rangle
\end{gathered}
$$

Therefore, the expression of the breakage kernel reads: 


$$
\Gamma(d)=C_{1} \frac{\sqrt{\left\langle[\Delta u(d)]^{2}\right\rangle}}{d} \exp \left(-C_{2} \frac{\sigma}{\rho_{d} d\left\langle[\Delta u(d)]^{2}\right\rangle}\right)
$$

Coulaloglou and Tavlarides expressed the coalescence kernel as the product of the collision frequency of two droplets, $h\left(v, v^{\prime}\right)$, and the collision efficiency, $\lambda\left(v, v^{\prime}\right)$. The collision frequency is based on the kinetic theory of gases:

$$
h\left(v, v^{\prime}\right)=C_{3} \sqrt{\left\langle[\Delta u(d)]^{2}\right\rangle+\left\langle\left[\Delta u\left(d^{\prime}\right)\right]^{2}\right\rangle}\left(v^{\frac{2}{3}}+v^{\prime \frac{2}{3}}\right)\left(v^{\frac{2}{9}}+v^{\prime \frac{2}{9}}\right)^{1 / 2}
$$

The collision efficiency, on the other hand, is based on the Shinnar theory [11], which considers that coalescence occurs if the contact time between the colliding droplets is long enough to drain the thin film trapped in between them:

$$
\lambda\left(v, v^{\prime}\right)=\exp \left(-\frac{C_{4} \mu_{c} \rho_{c}\left\langle\left[\Delta u\left(d+d^{\prime}\right)\right]^{2}\right\rangle^{\frac{3}{2}}}{\sigma^{2}}\left(\frac{v^{\frac{1}{3}} v^{\prime \frac{1}{3}}}{v^{\frac{1}{3}}+v^{\prime \frac{1}{3}}}\right)^{4}\right)
$$

Finally:

$$
\begin{aligned}
Q\left(v, v^{\prime}\right)=C_{3} \sqrt{\left\langle[\Delta u(d)]^{2}\right\rangle+\left\langle\left[\Delta u\left(d^{\prime}\right)\right]^{2}\right\rangle}\left(v^{\frac{2}{3}}\right. \\
\\
\left.\quad+v^{\prime \frac{2}{3}}\right)\left(v^{\frac{2}{9}}+v^{\prime} \frac{2}{9}\right)^{1 / 2} \exp \left(-\frac{C_{4} \mu_{c} \rho_{c}\left\langle\left[\Delta u\left(d+d^{\prime}\right)\right]^{2}\right\rangle^{\frac{3}{2}}}{\sigma^{2}}\left(\frac{v^{\frac{1}{3}} v^{\prime \frac{1}{3}}}{v^{\frac{1}{3}}+v^{\prime \frac{1}{3}}}\right)^{4}\right)
\end{aligned}
$$

As highlighted by Eq. (10) and (13), the breakage and coalescence kernels depend on the second-order structure function. As previously mentioned, in this work this function is computed according to Eq. (4), in which the adopted energy spectrum expression is the one proposed by Pope Eq. (5). It is worth remarking that, as in the original derivation of Coulaloglou and Tavlarides, the proposed generalized model presents four constant parameters $\left(C_{1}, C_{2}, C_{3}, C_{4}\right)$ that are experimentally determined since they include all the modeling uncertainties $[1,14,18]$. 


\section{Materials and methods}

\subsection{Materials}

To validate the proposed model, liquid-liquid dispersion experiments were carried out at different dispersed phase volume fractions, and, more important, at different continuous phase viscosities. The increase in the viscosity leads to a bigger Kolmogorov microscale, and therefore, to a wider dissipation range.

Distilled water was employed as the dispersed phase, while the continuous phase was composed of different mixtures of Isane 175 and Marcol 82. Isane 175 is an isoparaffanic solvent provided by Total, which assures a high purity of the product, having less than 20 ppm of aromatic compound and $1 \mathrm{ppm}$ of benzene. Marcol 82 is a mixture of liquid saturated hydrocarbons provided by ExxonMobil. Isane 175 and water have similar viscosities, whereas Marcol 82 has a much higher viscosity. Therefore, the two oils were mixed at different volume fractions in order to achieve various continuous phase viscosities (Table 1).

The experiments were performed in a stirred tank reactor (STR) of 1L volume, equipped with a 3-blades Mixel TT impeller, and 4 baffles to avoid vortex formation. The baffles have a width of $15 \mathrm{~mm}(\mathrm{~T} / 8)$ and a thickness of $5 \mathrm{~mm}$. The STR has a height, $\mathrm{H}$, of $120 \mathrm{~mm}$, and a diameter of the same size $(\mathrm{T}=\mathrm{H})$. The impeller diameter, $\mathrm{D}$, is half of the tank one $(\mathrm{D}=\mathrm{T} / 2)$. It is located at $\mathrm{H} / 3(40 \mathrm{~mm})$ from the bottom of the reactor (Fig. 2).

In order to monitor the time evolution of the droplet size distribution (DSD), a photooptical endoscopic probe provided by Sopat ${ }^{\circledR}$ was inserted in the reactor. This device generated flashes of light that, due to the different refraction indexes of the two phases, allowed to capture images of droplets with a size bigger than $20 \mu \mathrm{m}$ [23]. The collected images were then treated (Fig. 3) and analyzed through the software provided by the vendor to obtain the DSD and then the Sauter mean diameter. 


\subsection{Experimental procedure}

The experiments were performed at different oil viscosities (Table 1), dispersed phase volume fractions (1-2\%), and impeller rotation speeds $(600,700$ and $800 \mathrm{rpm})$.

The experimental procedure consists in filling the reactor with the desired fraction of the organic mixture and water. Then, stirring is started and the water-in-organic phase dispersion is created. After ten minutes of stirring, a first set of images is collected, representing the initial distribution of the experiment. Then, other sets of images are acquired, at regular time intervals, in order to assess the evolution of the DSD. Each acquisition lasted 60 seconds, with an image capturing rate of $5 \mathrm{~Hz}$, for a total of 300 images. In average, each picture presented 10 droplets.

Since the interfacial area is the property of interest, the Sauter mean diameter was determined for each experimental DSD. The Sauter mean diameter is defined as the ratio between the third and the second moment of the distribution:

$$
d_{32}=\frac{\int_{0}^{\infty} d^{3} n(d) \mathrm{d} d}{\int_{0}^{\infty} d^{2} n(d) \mathrm{d} d}
$$

In a closed stirred tank reactor, the total volume of the droplets is constant (so is the third-order moment with respect to droplet size), while the surface changes continuously (and so the second-order moment of the distribution). Therefore, the higher the interfacial area, the smaller the Sauter mean diameter and vice versa.

The Sauter mean diameter of the droplet distribution decreases by a few tens of micrometers per hour in each experiment. Therefore, during the sixty seconds of the acquisition procedure, the droplet size distribution was considered in a quasi-steady state and the Sauter mean diameter constant.

\subsection{OD Population Balance Model}

The general expression for the PBE depending on spatial coordinates (also called external) $\boldsymbol{x}$, internal coordinates $\boldsymbol{\varphi}$ and time $t$ is the following [5]: 


$$
\begin{gathered}
\frac{\partial n(\boldsymbol{x}, \boldsymbol{\varphi}, t)}{\partial t}+\left\langle u_{i}\right\rangle \frac{\partial n(\boldsymbol{x}, \boldsymbol{\varphi}, t)}{\partial x_{i}}-\frac{\partial}{\partial x_{i}}\left[D_{t} \frac{\partial n(\boldsymbol{x}, \boldsymbol{\varphi}, t)}{\partial x_{i}}\right] \\
=\frac{\partial}{\partial \varphi_{j}}\left[n(\boldsymbol{x}, \boldsymbol{\varphi}, t) \dot{\varphi}_{J}\right]+s(\boldsymbol{x}, \boldsymbol{\varphi}, t) .
\end{gathered}
$$

In this work, the volume of the droplets $v$ (and hence the size of the droplets) will be the only tracked internal coordinate $(\boldsymbol{\varphi}=\boldsymbol{v})$. The r.h.s. term of the equation, $s(\boldsymbol{x}, \boldsymbol{v}, t)$, represents the introduction or the loss of droplets due to breakage and coalescence:

$$
\begin{gathered}
s(\boldsymbol{x}, v, t)=s_{B R E A K}(\boldsymbol{x}, v, t)+s_{C O A L}(\boldsymbol{x}, v, t) \\
s_{B R E A K}(\boldsymbol{x}, v, t)=\int_{v}^{\infty} p\left(v^{\prime}\right) \beta\left(v \mid v^{\prime}\right) \Gamma\left(\boldsymbol{x}, v^{\prime}\right) n\left(\boldsymbol{x}, v^{\prime}, t\right) d v^{\prime}-\Gamma(\boldsymbol{x}, v) n(\boldsymbol{x}, v, t) \\
s_{C O A L}(\boldsymbol{x}, v, t)=\frac{1}{2} \int_{0}^{v} Q\left(\boldsymbol{x}, v-v^{\prime}, v^{\prime}\right) n\left(\boldsymbol{x}, v-v^{\prime}, t\right) n\left(\boldsymbol{x}, v^{\prime}, t\right) d v^{\prime} \\
-n(\boldsymbol{x}, v, t) \int_{0}^{\infty} Q\left(\boldsymbol{x}, v, v^{\prime}\right) n\left(\boldsymbol{x}, v^{\prime}, t\right) d v^{\prime}
\end{gathered}
$$

where $\Gamma$ and $Q$ are the breakage and coalescence kernels. $\beta\left(v \mid v^{\prime}\right)$ represents the daughter size distribution function (DDF), whose expression gives the probability that a mother droplet of volume $v^{\prime}$ breaks in two daughter droplets of volume $v$ and $v^{\prime}-v$. According to Coulaloglou and Tavlarides, the DDF proposed by Valentas and Amundson [24] has been employed. This function predicts the equi-sized breakage by being the most probable event, as experimentally confirmed by Andersson and Andersson [25].

In order to get a simplified OD model (where the OD denotes a non-dependency on spatial coordinates), the number density function is assumed to be homogeneous in the stirred tank reactor and defined as:

$$
\bar{n}(v, t)=\frac{1}{V} \int_{V} n(\boldsymbol{x}, v, t) d \boldsymbol{x}
$$


Substituting Eq. (16) into Eq. (15) and later applying a volume-average procedure, the following $0 \mathrm{D}$ model is obtained:

$$
\begin{gathered}
\frac{\partial \bar{n}(v, t)}{\partial t}=\int_{v}^{\infty} \beta\left(v \mid v^{\prime}\right) \frac{1}{V} \int_{x} \Gamma(\boldsymbol{x}, v) d \boldsymbol{x} \bar{n}\left(v^{\prime}, t\right) d v^{\prime}-\bar{n}(v, t) \int_{0}^{\infty} \frac{1}{V} \int_{\boldsymbol{x}} \mathrm{Q}\left(\boldsymbol{x}, v, v^{\prime}\right) d \boldsymbol{x} \bar{n}\left(v^{\prime}, t\right) d v^{\prime} \\
-\bar{n}(v, t) \frac{1}{V} \int_{\boldsymbol{x}} \Gamma(\boldsymbol{x}, v) d \boldsymbol{x}+\frac{1}{2} \int_{0}^{v} \frac{1}{V} \int_{\boldsymbol{x}} \mathrm{Q}\left(\boldsymbol{x}, v-v^{\prime}, v^{\prime}\right) d \boldsymbol{x} \bar{n}\left(v-v^{\prime}, t\right) \bar{n}\left(v^{\prime}, t\right) d v^{\prime}
\end{gathered}
$$

Although the volume-average procedure, the system is still dependent on the spatial coordinates, because the turbulent dissipation rate distribution inside the vessel is strongly inhomogeneous. Therefore, in this work, volume-average kernels are considered [19]:

$$
\begin{gathered}
\bar{\Gamma}(v)=\frac{1}{V} \int_{V} \Gamma(\boldsymbol{x}, v) d \boldsymbol{x}=\int_{0}^{\infty} \Gamma(\varepsilon, v) f(\varepsilon) d \varepsilon \\
\bar{Q}\left(v, v^{\prime}\right)=\frac{1}{V} \int_{V} Q\left(\boldsymbol{x}, v, v^{\prime}\right) d \boldsymbol{x}=\int_{0}^{\infty} Q\left(\varepsilon, v, v^{\prime}\right) f(\varepsilon) d \varepsilon
\end{gathered}
$$

where $f(\varepsilon)$ is the volumetric probability density function (pdf) of the turbulent kinetic energy dissipation rate $\varepsilon$. This function represents the probability density that an infinitesimal volume of the liquid-liquid contactor experiences a turbulent dissipation range in the range $[\varepsilon, \varepsilon+d \varepsilon]$. The pdf of $\varepsilon$ can be obtained running single-phase CFD simulations (since the investigated systems are diluted) of the liquid-liquid contactor in working conditions [20].

The Fixed Pivot Method, proposed by Kumar and Ramkrishna [26], was employed to solve the OD PBE. The size domain is discretized in 40 arithmetic intervals $\left[v_{i}, v_{i+1}\right]$. For each interval, a characteristic size $p_{i}$, called pivot, is defined $\left(v_{i}<p_{i}<v_{i+1}\right)$. Kumar and Ramkrishna postulated that all the droplets lying in the size interval $\left[v_{i}, v_{i+1}\right]$ have a size equal to the pivotal one, $p_{i}$. Based on this assumption, the NDF is expressed as:

$$
n(v, t)=\sum_{i=1}^{M} N_{i}(t) \delta\left(v-p_{i}\right)
$$


Substituting Eq. (19) in Eq.(16), Kumar and Ramkrishna derived the following expression:

$$
\begin{aligned}
\frac{d N_{i}(t)}{d t}= & \sum_{\substack{j, k \\
p_{i-1} \leq\left(p_{j}+p_{k}\right) \leq p_{i+1}}}^{j \geq k}\left(1-\frac{1}{2} \delta_{j, k}\right) \eta Q_{j, k} N_{j}(t) N_{k}(t)-\Gamma_{i} N_{i}(t) \\
& -N_{i}(t) \sum_{k=1}^{M} Q_{i, k} N_{k}(t)+\sum_{k=1}^{M} m_{i, k} \Gamma_{k} N_{k}(t)
\end{aligned}
$$

More details on this method can be found in our previous work [20].

\subsection{Numerical details}

While the consideration of the entire energy spectrum of the turbulence allows a more refined description of the physics of the breakage and coalescence events, the volumeaverage kernel formulation [19] permits to better account for the turbulent inhomogeneity than using a volume-averaged turbulent dissipation rate [20]. The coupling of the two methods, therefore, allows to tackle two of the most insidious problems in the PBE model, with acceptable computation time.

Single-phase CFD simulations of the experimental stirred tank reactor were carried out for each experimental impeller rotation speed and each continuous phase viscosity. The $k-\varepsilon$ two-equation turbulence model was employed with an enhanced wall-treatment function. More information on the reactor mesh and the adopted discretization schemes have been reported in a previous work [20]. Each simulation took averagely 4 to 5 hours on an Intel Xeon dual-core machine $(3.20 \mathrm{GHz})$ with $64 \mathrm{~GB}$ of RAM. From each simulation, the probability density function of $\varepsilon$ was extracted as a histogram constituted by 10000 bins. Each bin represented the probability of having a value of $\varepsilon$ in the stirred tank reactor between $\varepsilon_{i}$ and $\varepsilon_{i+1}$. For each interval $\left[\varepsilon_{i}, \varepsilon_{i+1}\right]$, the average $\varepsilon$ was computed and employed in Eq. (5), which was later substituted in Eq. (4) to compute the secondorder structure as a function of the droplet diameter. The integral of Eq. (4) was computed 
through the MATLAB function integral. This operation was repeated for each pivot of the PBE discretization (40 intervals) and the computed values were saved for later use in the PBE solution ("generalized model"). For each $\varepsilon$, the constants the Pope energy spectrum were found by solving the non-linear system of equations Eq. (6) with the MATLAB function fsolve, based on the Trust-Region Dogleg method. This first computation was run in MATLAB and took 15-20 minutes on an Intel Xeon dual-core machine $(3.20 \mathrm{GHz})$ with 64GB of RAM. Finally, through the second-order structure functions computed previously, the MATLAB routine took approximately 1 minute to solve the PBE for simulating 4 hours of real time. A schematic representation of the numerical procedure is given in Fig. 4.

\section{Results and discussions}

As previously mentioned, the four constants of the proposed coalescence and breakage kernels need to be found for the system under investigation. Therefore, parameters identification was first performed using a numerical optimization procedure, based on a given set of experimental data. Then, using these parameters, the "generalized" model has been tested using different experiments at increasing viscosity. The model performances were compared with the ones obtained with the Coulaloglou and Tavlarides kernels employed in a previous work [20] (the "initial" model), that considers the droplets size in the inertial subrange. In both cases, the turbulent inhomogeneity in the stirred tank reactor was accounted through the volumetric probability density function of the turbulent dissipation rate, which allowed the computation of volume-average kernels [19,20].

\subsection{Parameters identification}

The water-in-Isane experiments with $1 \%$ (taken from a previous work, [20]) and $2 \%$ (Fig. 5) of water volume fraction, and an impeller rotation speed of $600 \mathrm{rpm}$ (red circles), were used to identify the parameters. 
The optimization was performed in MATLAB using the patternsearch function (Global Optimization toolbox) to minimize the sum of the square of the differences between the experimental and simulated Sauter mean diameters (Fig. 6). The adjusted parameters (Table 2) were identified with an average relative error lower than $1 \%$ (Table 3). The relative errors were computed according to the following expression:

$$
\text { Relative Error }(\%)=100 \frac{\mid \text { experimental data }(t)-\text { simulated value }(t) \mid}{\text { experimental data }(t)}
$$

\subsection{Low continuous phase viscosity}

The generalized model was tested with experiments at low continuous phase viscosity (1.2 $\mathrm{cP}$ ), employing pure Isane (continuous phase) and water (dispersed phase, 1-2\% volume fraction), at different impeller rotation speeds (700 and 800rpm).

As can be seen in Fig. 7, the "generalized model" is capable of well reproducing the time evolution of the Sauter mean diameters both for different stirring conditions (i.e., different turbulent conditions) and disperse phase volume fractions. As already evidenced in our previous work [20], also the "initial model" can correctly reproduce the experimental $\mathrm{d}_{32}$ evolution. Indeed, the two models fit the experimental data with average relative errors below 3\% (Table 4).

It is interesting to remark that, in the considered continuous phase viscosity range, the boundaries between the inertial and dissipation subranges under moderated turbulent dissipation rates are in the order of few millimeters (as reported in Table 5). Therefore, even in the system with the lowest value of continuous phase viscosity considered here, a large number of droplets is contained in the dissipation range, since the majority of the droplets have dimensions below $700 \mu \mathrm{m}$ (Fig. 8). The reason why the "initial model" is however able to predict the experimental behavior could be due to the summation of two errors canceling out: on one side, the overestimation of the eddy turbulent kinetic energy (as discussed in Sec. 2.1), and, on the other side, the neglecting of the enstrophy 
contribution. Indeed, the "initial model" is based on the Kolmogorov second-order structure function, which only considers the turbulent kinetic energy of eddies with size smaller than the droplet diameter, assuming they all belong to the inertial subrange. On the other hand, the refined second-order structure function proposed by Davidson [2] depends both on the turbulent kinetic energy of eddies with size smaller than the droplet diameter and on the enstrophy of the bigger ones. While the two expressions give similar results in the low viscosity system considered here, only the second one (i.e. the generalized model) is based on correct theoretical basis and gives proper predictions when the viscosity of the continuous phase increases, as will be shown in the following section.

\subsection{High continuous phase viscosity}

The results obtained with both the "generalized" and the "initial" models were compared with the experiments performed at higher continuous phase viscosities $(2.4 \mathrm{cP}$, and $4.1 \mathrm{cP})$, obtained by increasing the proportion of Marcol 82 in the mixture (from 30 to 45\%, Table 1). As it can be seen in Fig. 9 and 10, for these experiments, the observed dynamics of the droplet size evolution are slower than in the case of pure Isane (lower viscosity). Indeed, the higher the continuous phase viscosity, the wider the dissipation subrange (Table 5), and the subsequent reduction of the eddy energy. This lower energy leads to lower pressure and velocity fluctuations at the droplet surface, and hence to a decrease of the breakage rate.

The two models were first compared with the experiments at $1-2 \%$ volume fraction of the disperse phase (water) in the continuous phase (oil) at $2.4 \mathrm{cP}$ viscosity. As illustrated in Fig. 9 and 10, for both concentrations, the "generalized model" correctly reproduces the time evolution of the Sauter mean diameter, regardless of the stirring rate (i.e. the turbulent conditions). Indeed, the average relative error for each experiment is always below $3 \%$ (Table 6-7). On the other hand, the "initial model" predicts Sauter mean diameters that are always smaller than the experimental ones. For these cases, the average relative errors are 
almost $20 \%$ of the experimental data (Table 6-7). As previously discussed, using the Kolmogorov second-order structure function results in an overestimation of the eddy turbulent kinetic energy in the dissipation subrange, which leads to higher breakage rates. This is consistent with the observed prediction of smaller droplets by the initial model.

The models were finally tested with the experiments performed using the continuous phase with the higher viscosity $(4.1 \mathrm{cP})$. Note that this was the maximum value of viscosity in order to achieve turbulent flow [27-30] at the lowest rotational speed experimentally investigated (Table 8). Again, the "generalized model" appears very efficient to predict the time-evolution of the Sauter mean diameter (see Fig. 11), with average relative errors below 2\% (Table 9). With the "initial" model the deviation between the measurements and the predictions is even larger than at $2.4 \mathrm{cP}$, since the average relative errors can go up to the $29 \%$ of the experimental data (Table 9). Indeed, the viscosity dissipative effect in the dissipation subrange is more important at $4.1 \mathrm{cP}$ than at $2.4 \mathrm{cP}$. This confirms the improvement provided by the generalized model, that implements a more appropriate and accurate calculation of the effective turbulent kinetic energy of eddies, and accounts for the effect of viscosity in the dissipation range.

\section{Conclusions}

In this work, we implemented an improved description of the turbulence spectrum in the breakage and coalescence kernels formulated by Coulaloglou and Tavlarides [1]. The Coulaloglou and Tavlarides kernels, as most of the kernels available in the literature for liquid-liquid dispersions, assume that the droplets have dimensions in the same range of the characteristic eddy sizes belonging to the inertial subrange of turbulence. This assumption is generally not valid in liquid-liquid extractors that work at moderated Re, and for which the boundaries between the inertial and the dissipation subrange have dimensions of millimeters. This assumption leads to even larger errors as the viscosity of 
the continuous phase increases. In these cases, the majority of the droplet sizes lies in the dissipation range, and the most adopted second-order structure function included in the most popular coalescence and breakage kernels, proposed by Kolmogorov for the inertial subrange, is not valid.

In this work, the Pope energy spectrum model [3] has been chosen to compute a generalized second-order structure function, valid in the whole turbulent energy spectrum $[2,15,16]$. The formulation proposed by Pope is indeed convenient, since it expresses 3 contributions, each one being predominant in a given energy range (i.e. the energycontaining, inertial, and dissipation subranges). Thanks to the inclusion of this generalized function, the breakage and coalescence rates of droplets having dimensions in the dissipation range can be taken into account, and this is particularly helpful for high viscosity systems.

To prove this last statement, a series of turbulent liquid-liquid dispersion experiments, considering increasing viscosity of the continuous phase (1.2, 2.1 and $4.2 \mathrm{cP})$, were performed in order to validate the proposed generalized kernels. The model was observed to perfectly reproduce the experimental evolution of the Sauter mean diameter, at low dispersed phase volume fraction, in the whole range of investigated viscosities and stirring rates.

Complementing previous works aimed at improving the description of the turbulent spectrum in liquid-liquid breakage kernels (Solsvik et al. [16], Karimi and Andersson [15]), these new results show that more accurate models can be derived. They rely on the combination of $i$ ) a refined description of the turbulence spectrum, using a proper secondorder velocity structure function, and ii) the consideration of turbulent dissipation heterogeneity in the apparatus, using the pdf of the turbulent dissipation rate $\varepsilon$. Improving the description of turbulence in coalescence and breakage kernels, at both the droplet and 
the apparatus scales, is indeed a strong requirement for model-based industrial liquid-liquid process development. 


\section{Notation}

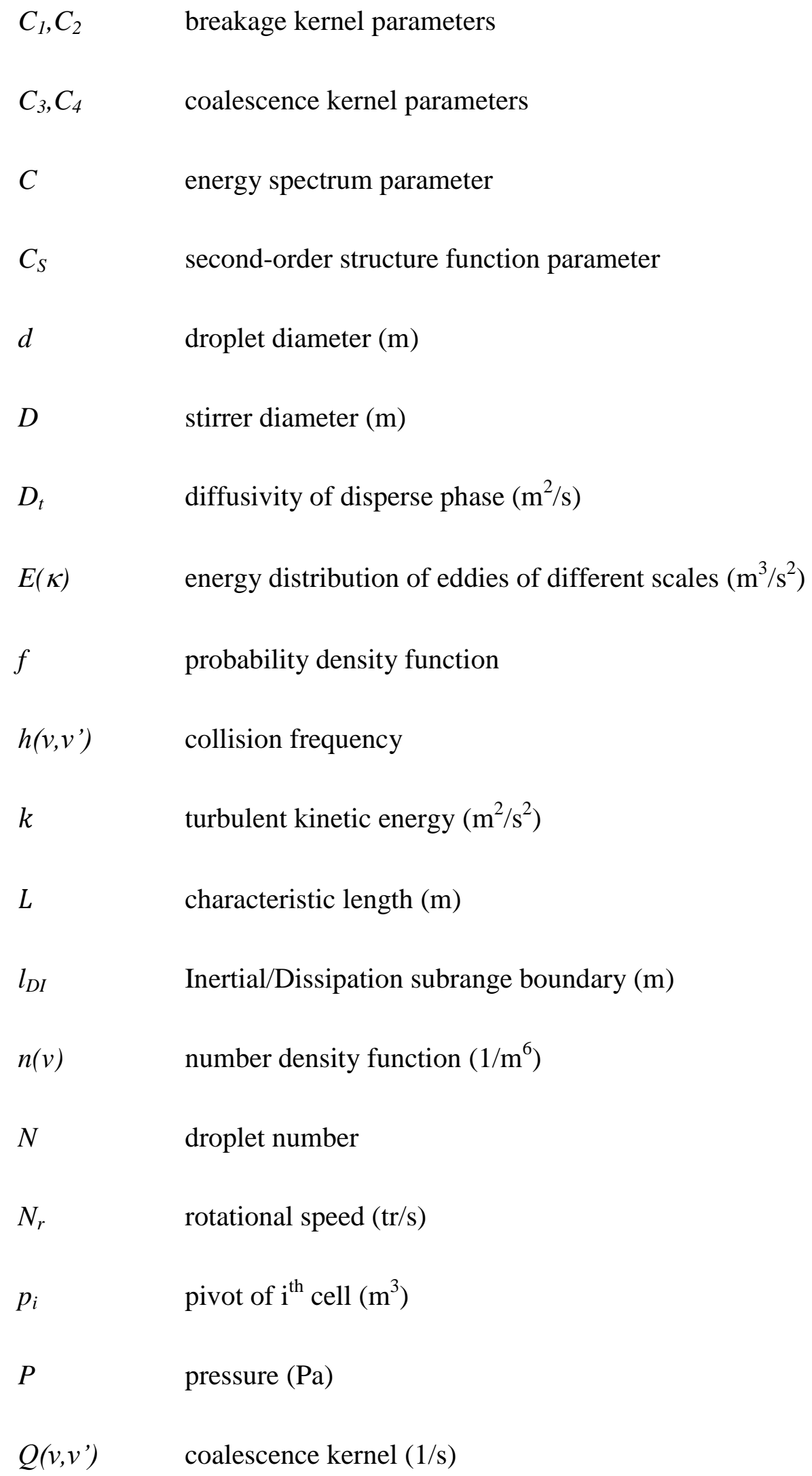




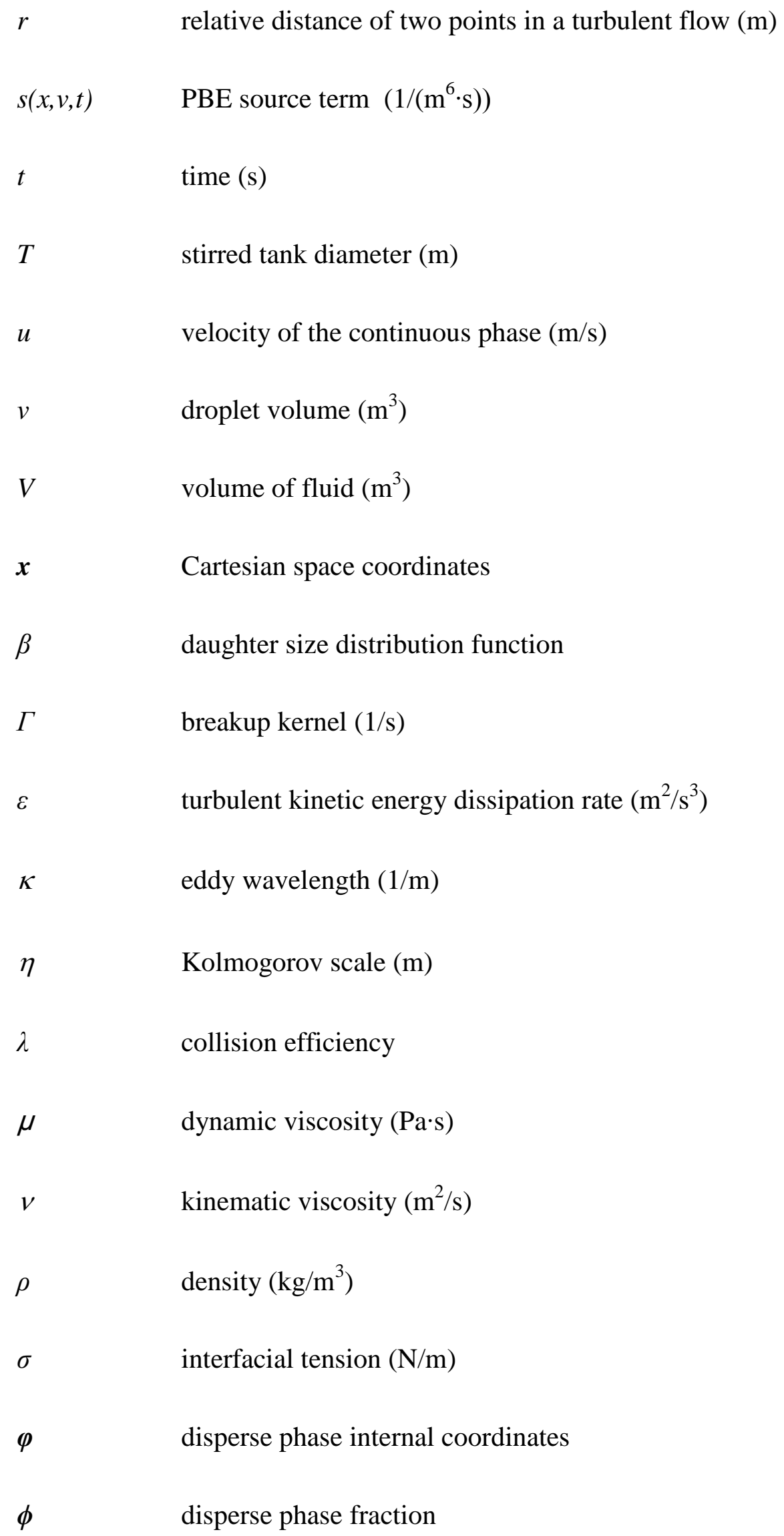


Acknowledgments

This work was founded by the Nuclear Energy Division of CEA (program DISN/PRATA). 


\section{References}

[1] C.A. Coulaloglou, L.L. Tavlarides, Description of interaction processes in agitated liquid-liquid dispersions, Chem. Eng. Sci. 32 (1977) 1289-1297. doi:10.1016/00092509(77)85023-9.

[2] P.A. Davidson, Turbulence: an introduction for scientists and engineers, Oxford University Press, Oxford, UK ; New York, 2004.

[3] S.B. Pope, Turbulent flows, Cambridge University Press, Cambridge ; New York, 2000 .

[4] D. Ramkrishna, Population balances: theory and applications to particulate systems in engineering, Academic Press, San Diego, CA, 2000.

[5] D.L. Marchisio, R.O. Fox, Computational Models for Polydisperse Particulate and Multiphase Systems, Cambridge University Press, Cambridge, 2013. doi:10.1017/CBO9781139016599.

[6] J. Baldyga, W. Podgórska, Drop break-up in intermittent turbulence: Maximum stable and transient sizes of drops, Can. J. Chem. Eng. 76 (1998) 456-470. doi:10.1002/cjce.5450760316.

[7] C.A. Coulaloglou, L.L. Tavlarides, Description of interaction processes in agitated liquid-liquid dispersions, Chem. Eng. Sci. 32 (1977) 1289-1297. doi:10.1016/00092509(77)85023-9.

[8] C. MartíNez-BazáN, J.L. MontañéS, J.C. Lasheras, On the breakup of an air bubble injected into a fully developed turbulent flow. Part 1. Breakup frequency, J. Fluid Mech. 401 (1999) 157-182. doi:10.1017/S0022112099006680.

[9] H. Luo, H.F. Svendsen, Theoretical model for drop and bubble breakup in turbulent dispersions, AIChE J. 42 (1996) 1225-1233. doi:10.1002/aic.690420505.

[10] J.O. Hinze, Fundamentals of the hydrodynamic mechanism of splitting in dispersion processes, AIChE J. 1 (1955) 289-295. doi:10.1002/aic.690010303.

[11] R. Shinnar, J.M. Church, Statistical Theories of Turbulence in Predicting Particle Size in Agitated Dispersions, Ind. Eng. Chem. 52 (1960) 253-256. doi:10.1021/ie50603a036.

[12] V. Alopaeus, J. Koskinen, K. I. Keskinen, J. Majander, Simulation of the population balances for liquid-liquid systems in a nonideal stirred tank. Part 2-parameter fitting and the use of the multiblock model for dense dispersions, Chem. Eng. Sci. 57 (2002) 1815-1825. doi:10.1016/S0009-2509(02)00067-2.

[13] R.V. Calabrese, T.P.K. Chang, P.T. Dang, Drop breakup in turbulent stirred-tank contactors. Part I: Effect of dispersed-phase viscosity, AIChE J. 32 (1986) 657-666. doi:10.1002/aic.690320416.

[14] P.J. Becker, F. Puel, R. Henry, N. Sheibat-Othman, Investigation of Discrete Population Balance Models and Breakage Kernels for Dilute Emulsification Systems, Ind. Eng. Chem. Res. 50 (2011) 11358-11374. doi:10.1021/ie2006033.

[15] M. Karimi, R. Andersson, An exploratory study on fluid particles breakup rate models for the entire spectrum of turbulent energy, Chem. Eng. Sci. 192 (2018) 850863. doi:10.1016/j.ces.2018.08.016. 
[16] J. Solsvik, H.A. Jakobsen, A review of the statistical turbulence theory required extending the population balance closure models to the entire spectrum of turbulence, AIChE J. 62 (2016) 1795-1820. doi:10.1002/aic.15128.

[17] J. Solsvik, H.A. Jakobsen, Development of Fluid Particle Breakup and Coalescence Closure Models for the Complete Energy Spectrum of Isotropic Turbulence, Ind. Eng. Chem. Res. 55 (2016) 1449-1460. doi:10.1021/acs.iecr.5b04077.

[18] A. Amokrane, S. Charton, N. Sheibat-Othman, J. Becker, J.P. Klein, F. Puel, Development of a CFD-PBE coupled model for the simulation of the drops behaviour in a pulsed column, Can. J. Chem. Eng. 92 (2014) 220-233. doi:10.1002/cjce.21933.

[19] A. Buffo, J. De Bona, M. Vanni, D.L. Marchisio, Simplified volume-averaged models for liquid-liquid dispersions: Correct derivation and comparison with other approaches, Chem. Eng. Sci. 153 (2016) 382-393. doi:10.1016/j.ces.2016.07.032.

[20] S. Castellano, N. Sheibat-Othman, D. Marchisio, A. Buffo, S. Charton, Description of droplet coalescence and breakup in emulsions through a homogeneous population balance model, Chem. Eng. J. 354 (2018) 1197-1207. doi:10.1016/j.cej.2018.07.176.

[21] S.G. Saddoughi, S.V. Veeravalli, Local isotropy in turbulent boundary layers at high Reynolds number, J. Fluid Mech. 268 (1994) 333. doi:10.1017/S0022112094001370.

[22] G.K. Batchelor, Diffusion in a field of homogeneous turbulence, Math. Proc. Camb. Philos. Soc. 48 (1952) 345-362. doi:10.1017/S0305004100027687.

[23] A. Amokrane, S. Maaß, F. Lamadie, F. Puel, S. Charton, On droplets size distribution in a pulsed column. Part I: In-situ measurements and corresponding CFD-PBE simulations, Chem. Eng. J. 296 (2016) 366-376. doi:10.1016/j.cej.2016.03.089.

[24] K.J. Valentas, N.R. Amundson, Breakage and Coalescence in Dispersed Phase Systems, Ind. Eng. Chem. Fundam. 5 (1966) 533-542. doi:10.1021/i160020a018.

[25] R. Andersson, B. Andersson, On the breakup of fluid particles in turbulent flows, AIChE J. 52 (2006) 2020-2030. doi:10.1002/aic.10831.

[26] S. Kumar, D. Ramkrishna, On the solution of population balance equations by discretization-I. A fixed pivot technique, Chem. Eng. Sci. 51 (1996) 1311-1332. doi:10.1016/0009-2509(96)88489-2.

[27] N. Harnby, M.F. Edwards, A.W. Nienow, eds., Mixing in the process industries, 2nd ed., pbk. ed, Butterworth-Heinemann, Oxford ; Boston, 1997.

[28] M. Jenne, M. Reuss, A critical assessment on the use of $\mathrm{k}-\varepsilon$ turbulence models for simulation of the turbulent liquid flow induced by a Rushton-turbine in baffled stirred-tank reactors, Chem. Eng. Sci. 54 (1999) 3921-3941. doi:10.1016/S00092509(99)00093-7.

[29] K. Van't Riet, J.M. Smith, The trailing vortex system produced by Rushton turbine agitators, Chem. Eng. Sci. 30 (1975) 1093-1105. doi:10.1016/0009-2509(75)870126.

[30] E.L. Paul, V.A. Atiemo-Obeng, S.M. Kresta, eds., Handbook of industrial mixing: science and practice, Wiley-Interscience, Hoboken, N.J, 2004. 


\section{Tables}

Table 1: Properties of Isane 175/Marcol 82 mixtures employed as solvents in the emulsification experiments

\begin{tabular}{|c|c|c|c|c|}
\hline \multicolumn{2}{|c|}{ Solvents } & \multicolumn{3}{|c|}{ Physical-chemical properties } \\
\hline Isane $[\%]$ & Marcol [\%] & 目(mPa.s) & $\rho\left(\mathrm{kg} / \mathrm{m}^{3}\right)$ & $\sigma(\mathbf{m N} / \mathbf{m})$ \\
\hline 100 & 0 & 1.219 & 757.4 & 38.30 \\
\hline 0 & 100 & 12.347 & 850.8 & 41.20 \\
\hline 70 & 30 & 2.429 & 783.4 & 38.21 \\
\hline 55 & 45 & 4.102 & 799.9 & 39.81 \\
\hline
\end{tabular}

Table 2: Identified parameters for the "generalized" model. Also the parameters for the "initial" model are reported [20]

\begin{tabular}{|c|cc|cc|}
\cline { 2 - 5 } \multicolumn{1}{c|}{} & \multicolumn{2}{c|}{ Breakup } & \multicolumn{2}{c|}{ Coalescence } \\
\cline { 2 - 5 } & $\mathbf{C}_{1}$ & $\mathbf{C}_{2}$ & $\mathbf{C}_{3}$ & $\mathbf{C}_{\mathbf{4}}$ \\
\hline “Generalized" & $2.23 \times 10^{-4}$ & $7.08 \times 10^{-1}$ & 1.0073 & $1.68 \times 10^{18}$ \\
\hline "Initial" & $1.20 \times 10^{-3}$ & $7.11 \times 10^{-1}$ & $1.95 \times 10^{-2}$ & $2.05 \times 10^{14}$ \\
\hline
\end{tabular}


Table 3: Relative errors according to Eq. (22) in the determination of the adjusted parameters at an impeller rotation speed of $600 \mathrm{rpm}$ :

\begin{tabular}{|c|c|c|c|c|c|c|}
\cline { 2 - 7 } \multicolumn{1}{|c|}{} & \multicolumn{3}{c|}{ 1\% water-in-Isane } & \multicolumn{3}{c|}{ 2\% water-in-Isane } \\
\hline Experimental $(\boldsymbol{\mu m})$ & 200.5 & 197.6 & 193.9 & 318.2 & 300.8 & 285.9 \\
\hline Optimization ( $\boldsymbol{\mu m})$ & 201.2 & 196.8 & 194.4 & 320.4 & 299.3 & 284.4 \\
\hline Relative error (\%) & $\mathbf{0 . 3 5}$ & $\mathbf{0 . 4 3}$ & $\mathbf{0 . 2 5}$ & $\mathbf{0 . 7 1}$ & $\mathbf{0 . 4 8}$ & $\mathbf{0 . 5 3}$ \\
\hline Average rel. err. (\%) & & & & & & \\
\hline
\end{tabular}

Table 4: Relative errors (Eq. (22)) by fitting the 1-2\% water-in-Isane experiments through the "generalized" and "initial" models:

\begin{tabular}{|c|c|c|c|c|c|c|c|c|c|c|c|c|}
\hline & \multicolumn{6}{|c|}{$1 \%$ water-in-Isane } & \multicolumn{6}{|c|}{$2 \%$ water-in-Isane } \\
\hline & \multicolumn{3}{|c|}{$700 \mathrm{rpm}$} & \multicolumn{3}{|c|}{800 rpm } & \multicolumn{3}{|c|}{$700 \mathrm{rpm}$} & \multicolumn{3}{|c|}{800 rpm } \\
\hline & \multicolumn{12}{|c|}{ “Generalized" model } \\
\hline Rel err. (\%) & 2.18 & 2.42 & 2.47 & 0.78 & 0.07 & 0.13 & 0.41 & 3.57 & 1.26 & 0.95 & 2.43 & 1.34 \\
\hline \multirow[t]{2}{*}{ Average (\%) } & \multicolumn{3}{|c|}{2.36} & \multicolumn{3}{|c|}{0.33} & \multicolumn{3}{|c|}{1.75} & \multicolumn{3}{|c|}{1.57} \\
\hline & \multicolumn{12}{|c|}{ "Initial" model } \\
\hline Rel err. (\%) & 1.35 & 1.92 & 0.26 & 0.07 & 1.18 & 0.34 & 2.98 & 0.19 & 4.28 & 0.95 & 0.98 & 0.81 \\
\hline Average (\%) & \multicolumn{3}{|c|}{1.18} & \multicolumn{3}{|c|}{0.53} & \multicolumn{3}{|c|}{2.48} & \multicolumn{3}{|c|}{0.91} \\
\hline
\end{tabular}


Table 5: Kolmogorov microscale $(\eta)$ and Inertial/Dissipation subranges boundary $\left(l_{\mathrm{DI}}\right)$ for the Isane/Marcol experiments at different viscosity and turbulent conditions

\begin{tabular}{c|c|ccc}
\hline \multirow{2}{*}{ Viscosity (cP) } & Turbulence & \multicolumn{3}{|c}{ Speed [rpm] / Average $\boldsymbol{\varepsilon}\left[\mathbf{m}^{2} / \mathbf{s}^{3}\right]$} \\
& scales & $600 / 0.62$ & $700 / 1.01$ & $800 / 1.42$ \\
\hline \multirow{2}{*}{1.2} & $\eta[? \mathrm{~m}]$ & 50.92 & 45.07 & 41.39 \\
& $1_{\mathrm{DI}}[\mathrm{mm}]$ & 3.05 & 2.70 & 2.48 \\
\hline \multirow{2}{*}{2.4} & $\eta[? \mathrm{~m}]$ & 82.94 & 73.89 & 67.1 \\
& $1_{\mathrm{DI}}[\mathrm{mm}]$ & 4.98 & 4.43 & 4.03 \\
\hline \multirow{2}{*}{4.1} & $\eta[? \mathrm{~m}]$ & 120.96 & 107.76 & 97.87 \\
& $1_{\mathrm{DI}}[\mathrm{mm}]$ & 7.26 & 6.47 & 5.87 \\
\end{tabular}

Table 6: Relative errors (Eq. (22)) by fitting the $1 \%$ water-in-Isane $70 \%$ Marcol30\% experiments through the "generalized" and "initial" models:

\begin{tabular}{|c|c|c|c|c|c|c|c|c|c|}
\hline & \multicolumn{3}{|c|}{600 rpm } & \multicolumn{3}{|c|}{$700 \mathrm{rpm}$} & \multicolumn{3}{|c|}{800 rpm } \\
\hline & \multicolumn{9}{|c|}{ “Generalized" model } \\
\hline Rel. err. (\%) & 2.46 & 0.48 & 2.46 & 1.27 & 2.35 & 0.65 & 2.07 & 1.06 & 3.00 \\
\hline Average (\%) & \multicolumn{3}{|c|}{1.80} & \multicolumn{3}{|c|}{1.42} & \multicolumn{3}{|c|}{2.04} \\
\hline & \multicolumn{9}{|c|}{ "Initial" model } \\
\hline Rel. err. (\%) & 5.66 & $\mathbf{1 7 . 1 7}$ & 19.12 & 15.85 & 25.57 & 25.52 & 16.56 & 20.79 & 26.67 \\
\hline Average (\%) & \multicolumn{3}{|c|}{13.98} & \multicolumn{3}{|c|}{22.31} & \multicolumn{3}{|c|}{21.34} \\
\hline
\end{tabular}

Table 7: Relative errors (Eq. (22)) by fitting the $2 \%$ water-in-Isane $70 \%$ Marcol30\% experiments through the "generalized" and "initial" models:

\begin{tabular}{|c|c|c|c|c|c|c|c|c|c|}
\hline & \multicolumn{3}{|c|}{600 rpm } & \multicolumn{3}{|c|}{700 rpm } & \multicolumn{3}{|c|}{800 rpm } \\
\hline & \multicolumn{9}{|c|}{ "Generalized" model } \\
\hline Rel. err. (\%) & 1.06 & 1.32 & 0.11 & 2.76 & 1.38 & 3.75 & 0.72 & 3.53 & 2.32 \\
\hline Average (\%) & \multicolumn{3}{|c|}{$\mathbf{0 . 8 3}$} & \multicolumn{3}{|c|}{2.63} & \multicolumn{3}{|c|}{2.19} \\
\hline & \multicolumn{9}{|c|}{ "Initial" model } \\
\hline Rel. err. (\%) & 13.55 & 18.76 & 20.59 & 11.86 & 21.40 & 29.55 & $\mathbf{1 0 . 5 0}$ & 24.16 & 27.58 \\
\hline Average (\%) & \multicolumn{3}{|c|}{17.63} & \multicolumn{3}{|c|}{20.94} & \multicolumn{3}{|c|}{20.74} \\
\hline
\end{tabular}


Table 8: Impeller Reynolds numbers at different rotational speeds and viscosities

\begin{tabular}{|c|c|c|c|}
\hline \multirow{2}{*}{$\begin{array}{c}\text { Rotational speed } \\
{[\mathrm{rpm}]}\end{array}$} & \multicolumn{3}{|c|}{ Reynolds number } \\
\hline & V $=1.2 \mathrm{cP}$ & T $=2.4 \mathrm{cP}$ & 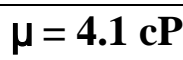 \\
\hline 600 & 22368 & 11611 & 7020 \\
\hline 700 & 26096 & 13546 & 8190 \\
\hline 800 & 29824 & 15481 & 9360 \\
\hline
\end{tabular}

Table 9: Relative errors (Eq. (22)) by fitting the 1\% water-in-Isane55\%Marcol45\% experiments through the "generalized" and "initial" models:

\begin{tabular}{|c|c|c|c|c|c|c|c|c|c|}
\hline & \multicolumn{3}{|c|}{$600 \mathrm{rpm}$} & \multicolumn{3}{|c|}{$700 \mathrm{rpm}$} & \multicolumn{3}{|c|}{800 rpm } \\
\hline & \multicolumn{9}{|c|}{ “Generalized" model } \\
\hline Rel. err. (\%) & 0.52 & 0.43 & 0.25 & 1.69 & 2.04 & 1.23 & 1.41 & 1.73 & 1.12 \\
\hline \multirow[t]{2}{*}{ Average (\%) } & \multicolumn{3}{|c|}{0.4} & \multicolumn{3}{|c|}{1.65} & \multicolumn{3}{|c|}{1.42} \\
\hline & \multicolumn{9}{|c|}{ "Initial" model } \\
\hline Rel. err. (\%) & 11.69 & 15.31 & 20.67 & 19.74 & 32.77 & 35.84 & 18.74 & 26.47 & 30.89 \\
\hline Average (\%) & \multicolumn{3}{|c|}{15.89} & \multicolumn{3}{|c|}{29.45} & \multicolumn{3}{|c|}{25.37} \\
\hline
\end{tabular}




\section{Figures}

Fig. 1: Energy spectrum representation of the eddies according to their wavelength in the energy-containing range and the inertial and dissipation subranges of turbulence.

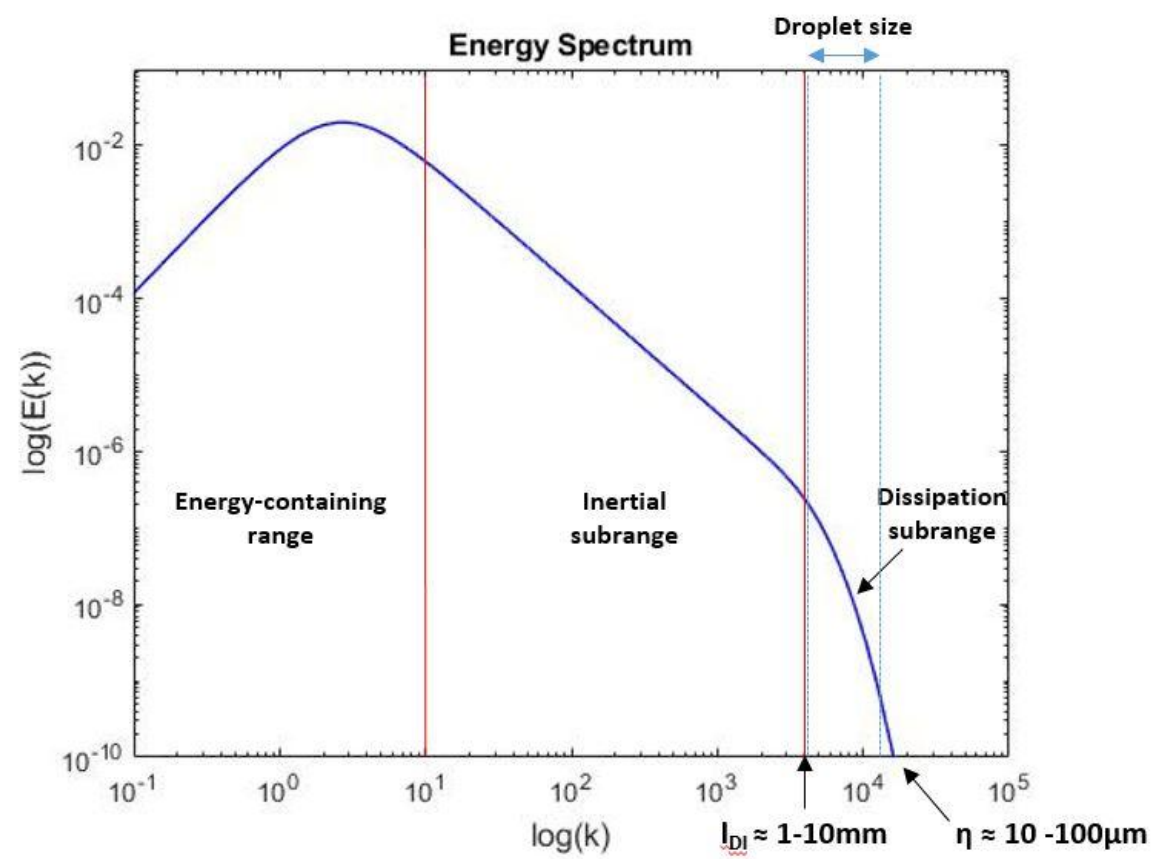

Fig. 2: Details of the geometry of the stirred tank reactor employed in the liquid-liquid dispersion experiments

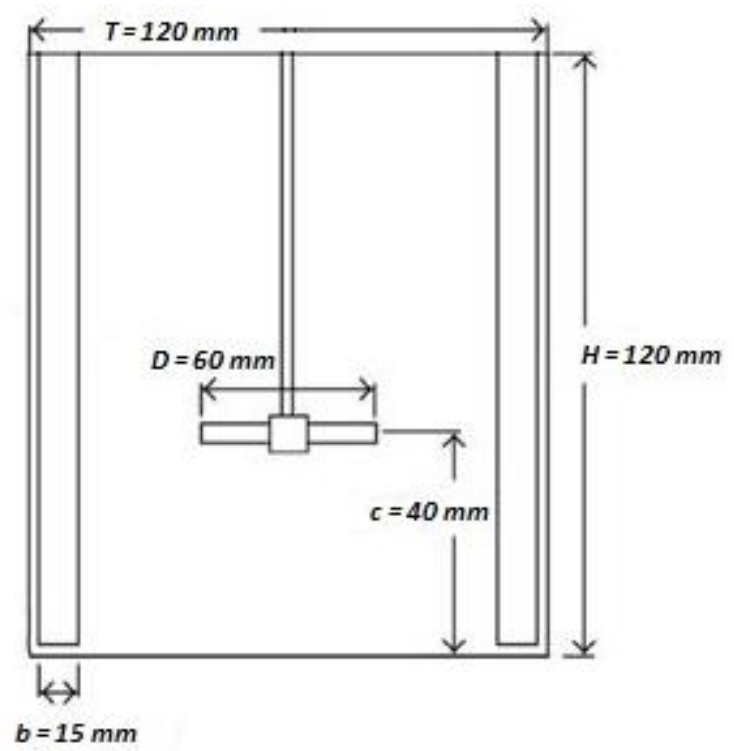


Fig. 3: Droplet caption with the SOPAT® probe (left) and detection (right, green circles)
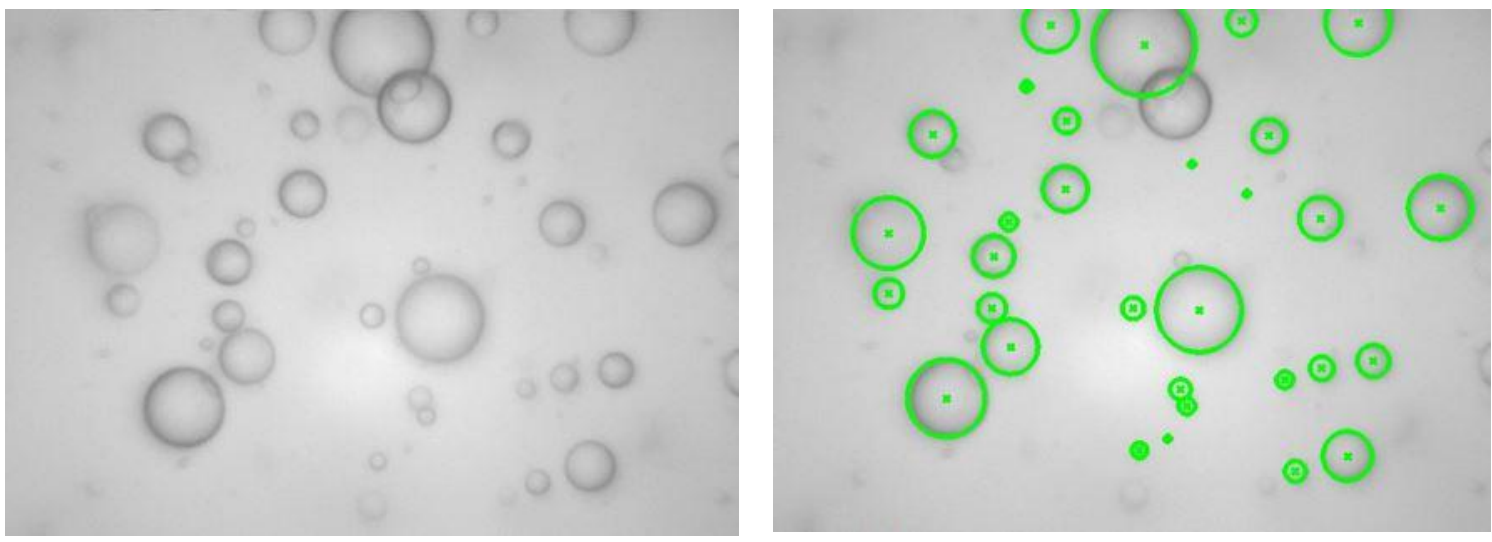

Fig. 4: Main steps and sequence of the numerical procedure

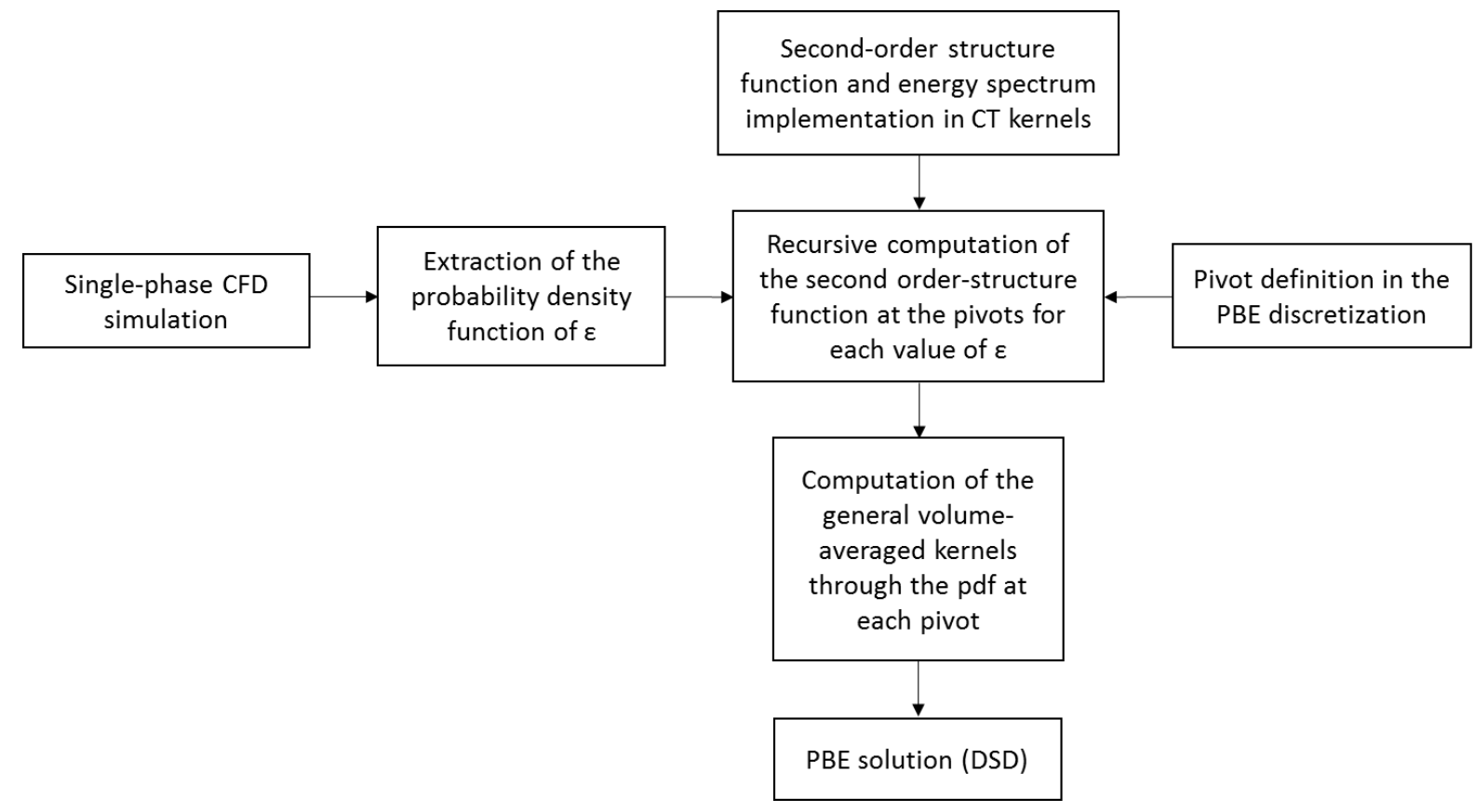


Fig. 5: Time-evolution of the droplet Sauter mean diameters for water-in-oil experiments: continuous phase viscosity $1.2 \mathrm{cP}$, water volume fraction of $2 \%$, stirring rate of 600,700 , and $800 \mathrm{rpm}$.

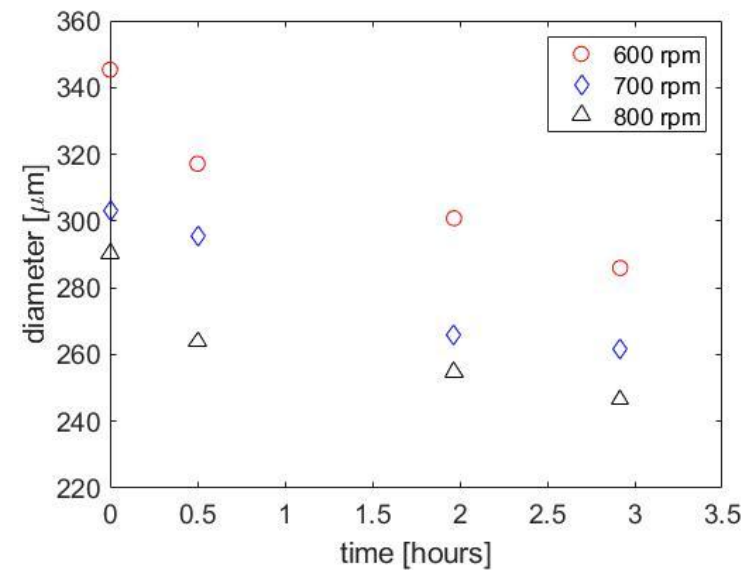

Fig. 6: Parameters identification of the "generalized model" on water-in-Isane experiments (denoted by an error bar of $1 \%$ the experimental value): continuous phase viscosity $1.2 \mathrm{cP}$, water volume fraction of 1 (left) -2 (right) $\%$, stirring rate of $600 \mathrm{rpm}$.
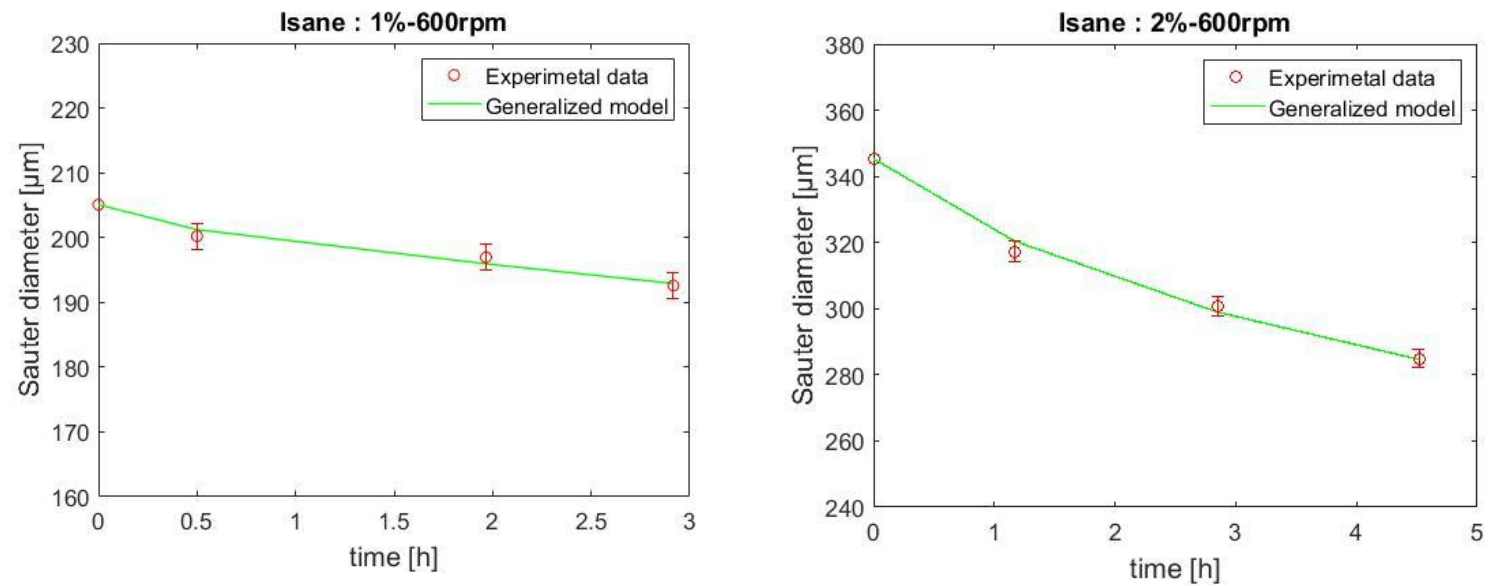
Fig. 7: "Generalized model" testing on water-in-Isane experiments (denoted by an error bar of $3 \%$ the experimental value): continuous phase viscosity $1.2 \mathrm{cP}$, water volume fraction of 1 (top) - 2 (bottom) \%, stirring rate of 700 (left) and 800 (right) rpm.
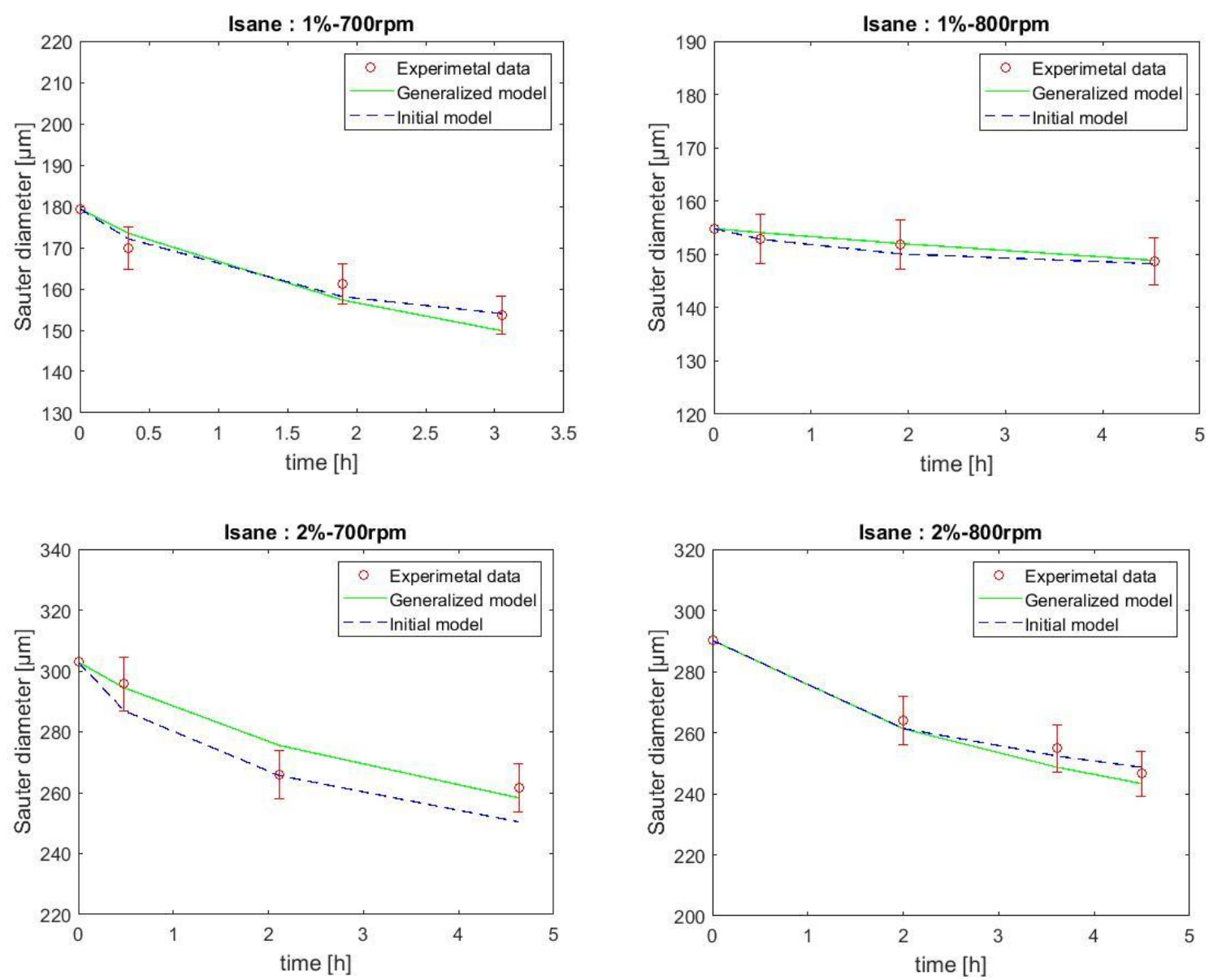

Fig. 8: Examples of droplet number density function for water-in-Isane experiments: water volume fraction of $2 \%$, stirring rate of 600 (green), 700 (red) and 800 (blue) rpm.

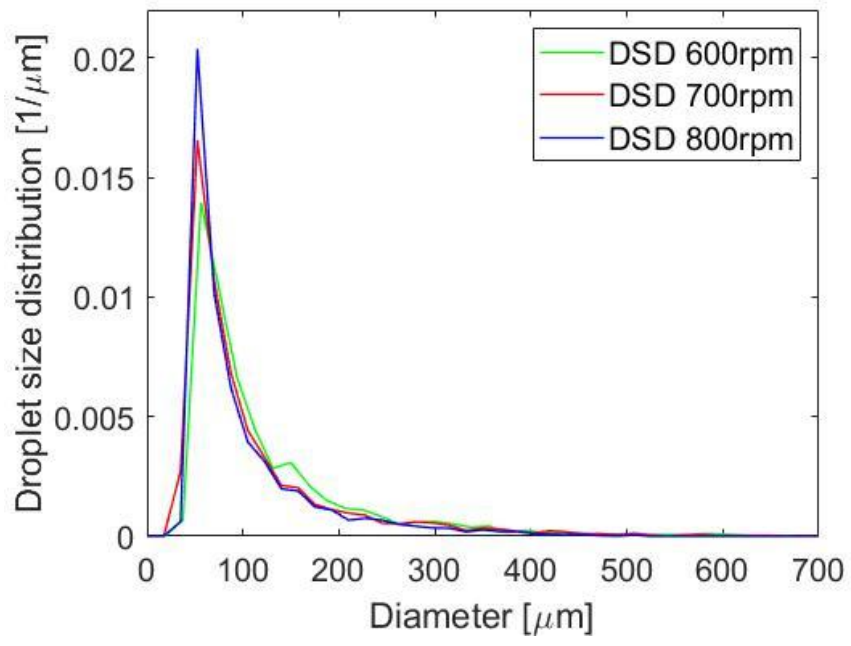


Fig. 9: "Generalized model" testing on water-in-Isane70\%/Marcol30\% experiments (denoted by an error bar of $3 \%$ the experimental value): continuous phase viscosity $2.4 \mathrm{cP}$, water volume fraction of $1 \%$, stirring rate of 600 (top-left), 700 (top-right), and 800 (bottom) rpm.
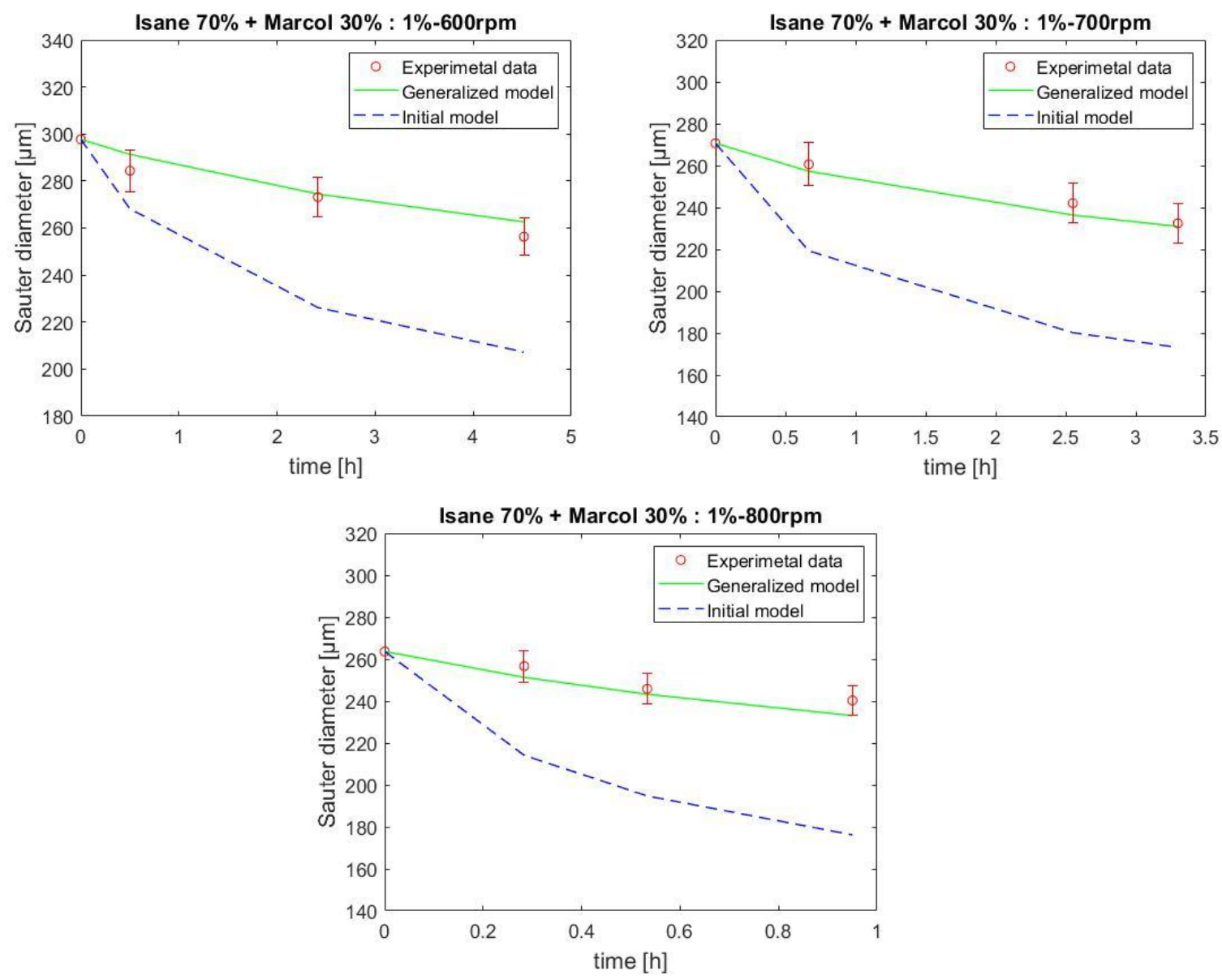
Fig. 10 "Generalized model" testing on water-in-Isane70\%/Marcol30\% experiments (denoted by an error bar of $3 \%$ the experimental value): continuous phase viscosity $2.4 \mathrm{cP}$, water volume fraction of $2 \%$, stirring rate of 600 (top-left), 700 (top-right), and 800 (bottom) rpm.
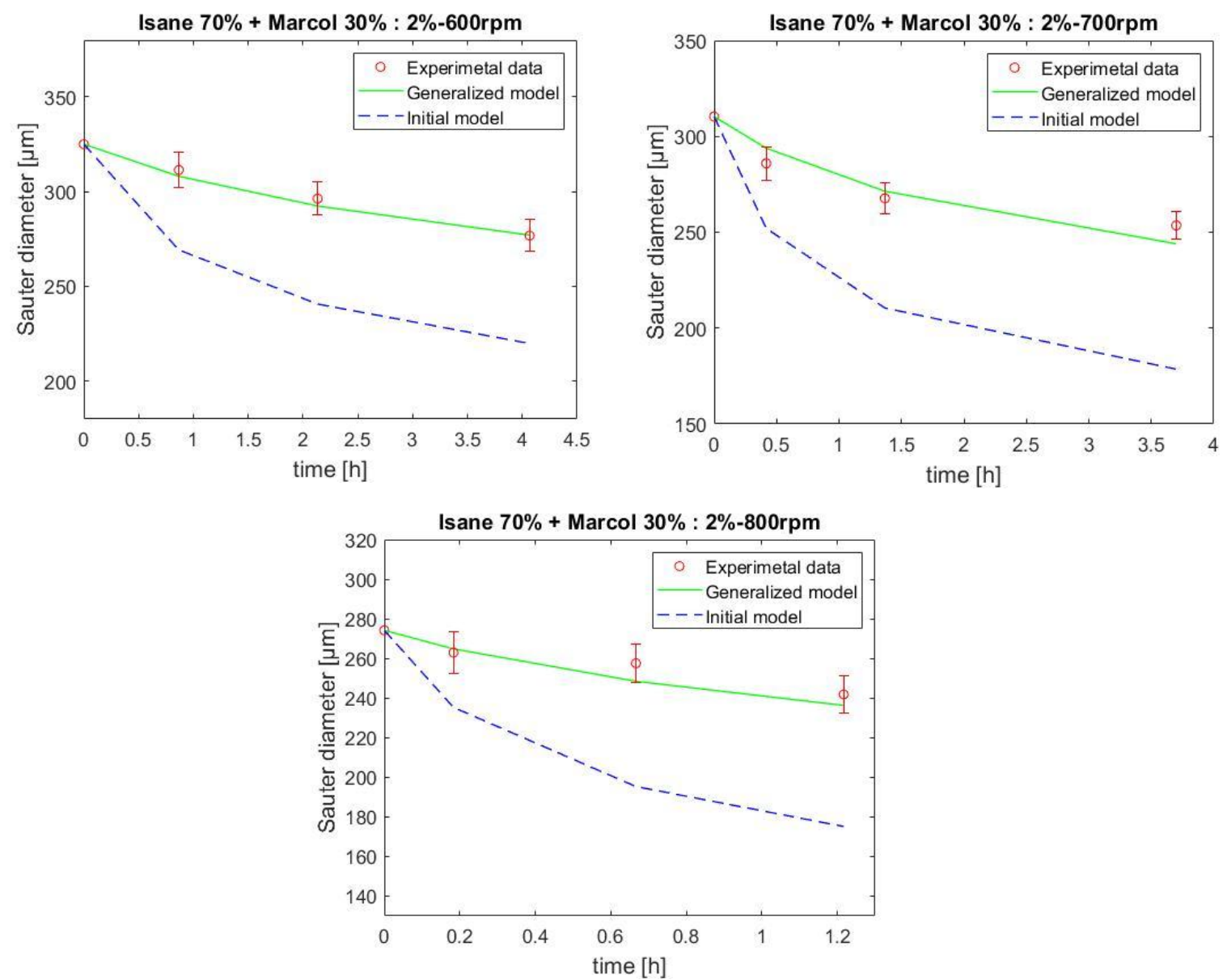
Fig. 11 "Generalized model" testing on water-in-Isane55\%/Marcol45\% experiments (denoted by an error bar of $3 \%$ the experimental value): continuous phase viscosity $4.1 \mathrm{cP}$, water volume fraction of $1 \%$, stirring rate of 600 (top-left), 700 (top-right), and 800 (bottom) rpm.
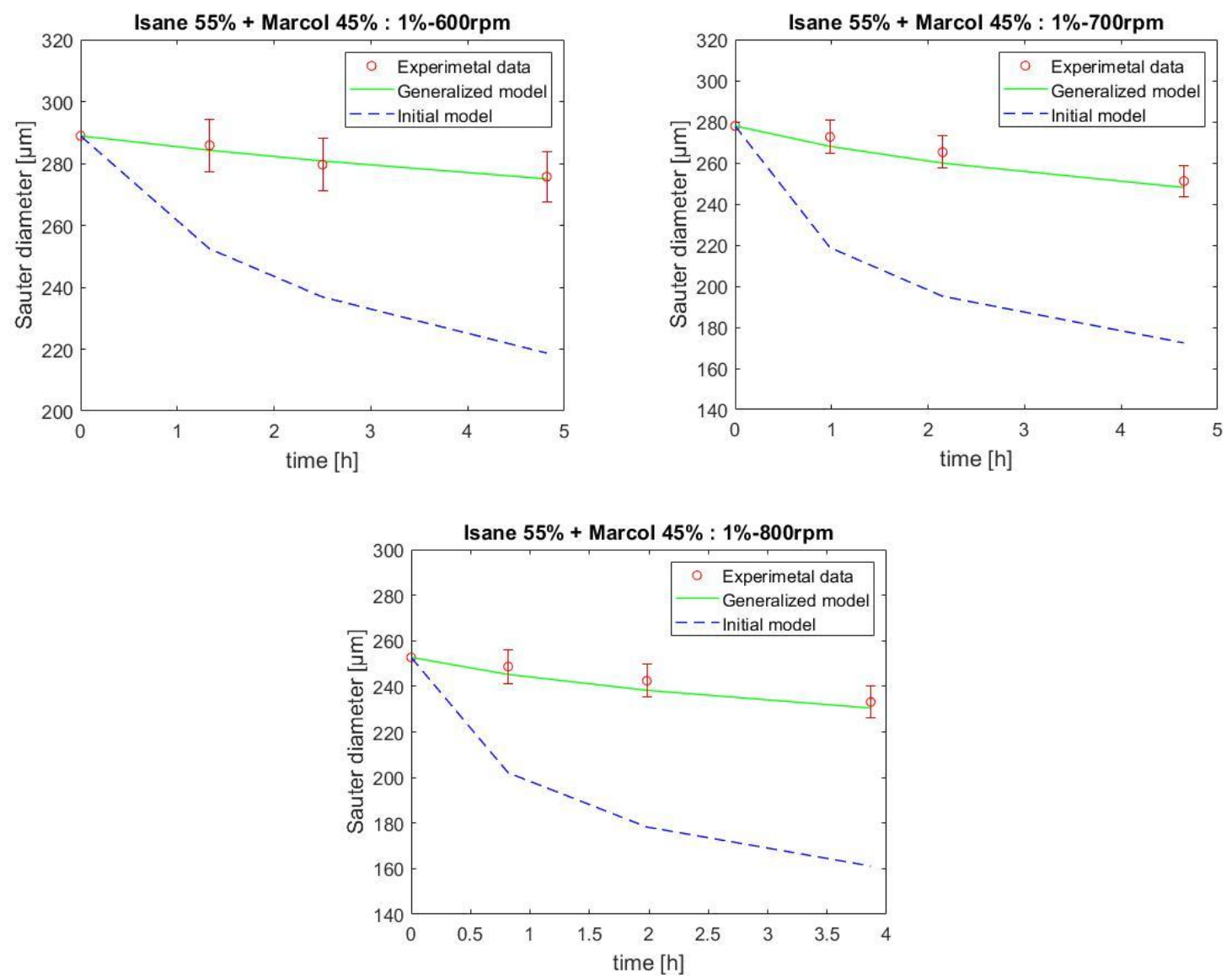